\title{
Marrow-Derived Mesenchymal Stem Cells Transfected with Survinvin Gene Protect Kidney from ischemia-reperfusion Injury in Rats
}

\section{Yang Xiaofeng}

Department of Urology, Zaozhuang Municipal Hospital

Qianqian Wang (D 58332365@qq.com )

Department of Nephrology, Zaozhuang Municipal Hospital

\section{Research Article}

Keywords: Bone marrow-derived stem cells, ischemic reperfusion injury, survinvin, rats

Posted Date: September 13th, 2021

DOl: https://doi.org/10.21203/rs.3.rs-895074/v1

License: (c) (1) This work is licensed under a Creative Commons Attribution 4.0 International License.

Read Full License 


\section{Abstract}

Objective: To investigate the survival ability of bone marrow Mesenchymal stem cells (MSCs) transfected with survinvin gene in the microenvironment of renal ischemia,and to study the ability and mechanism of repairing renal ischemia-reperfusion injury in rats.

Method: Mesenchymal stem cells (MSCs) from bone marrow of male Sprague-Dawley rat were infected with the self-inactive lentiviral vector and transfected with the Survinvin gene recombinant vector and then EGFP-tagged. After amplification and culture, they were detected by green fluorescence and then retained. 48 specific pathogen-free C57BL/6J mice were randomly divided into 4 groups of 12 each. Rats in the control group were only surgically exposed. The other 3 groups were surgically exposed and the bilateral renal arteries were clamped for 45 minutes to restore blood supply, and models of renal ischemia-reperfusion were established. There were control group,ischemia reperfusion group(Marked as IR group), empty virus transfection transplantation group(Marked as MSCs group) or survinvin gene transfection transplantation group(Marked as SVV/MSCs group), and sequentially injected with normal saline,normal saline, $1 \times 106$ MSCs infected with the self-inactive lentiviral vector or $1 \times 106$ survivin geneexpressing MSCs. At different time points of $1 d, 3 d, 7 d, 14 d$, collect serum to test blood urea nitrogen detection, to cut the rat kidney section for quantitative analysis, HE staining to observe renal issues changes and the degree of renal tubular damage and IL-10 by using ELISA detection.

Result: The MSCs with resuscitation and expansion culture had strong proliferation and good fluorescence. The creatinine urea nitrogen level in the MSCs group and SVV/MSCs group was significantly lower than that in the IR group and control group $(p<0.01$ or $p<0.05)$. The pathological damage score of HE staining in the kidney was lighter in the stem cell transplantation group, and the SVV/MSCs group was significantly lower than the other two groups $(p<0.01$ or $p<0.05)$. On the 3rd and 14th day, the number of transplanted cells in the kidney tissue was much higher in the SVV/MSCs group than in the MSCs group. The MSCs expressing EGFP were mainly distributed around the glomerulus, the small vessel inner wall, and the interstitial between the renal tubule and the renal tubule. However, MSCs expressing EGFP were hardly seen on the inner wall of the renal tubule. The levels of protective factors IL10 increased after renal ischemic injury. SVV/MSCs group was also significantly more than IR group or MSCs group $(P<0.01$ or $p<0.05)$. And there was no statistical difference from the normal control group on the 14 th day.

Conclusion: Transfection of Survinifin gene can increase the survival ability of MSCs in ischemic kidney. After transplantation, MSCs are not directly differentiated into injured tubular endothelial cells, which further promote the repair of kidney damage through its strong paracrine effect.

\section{Background}

Clinically, the incidence of acute renal failure (ARF) caused by ischemia-reperfusion injury is extremely high, and the short-term survival rate is extremely poor. However, there is no effective treatment method at 
present, which is an important problem that has always plagued clinicians.As a new possible treatment, the repair of renal ischemia-reperfusion injury by Bone Marrow-derived Mesenchymal Stem Cells (BMSCs) has been confirmed by more and more scholars $(1,2,3)$. This brings new hope for clinical treatment of acute renal failure. Previously, it was thought that transplanted MSCs could transform into damaged cells, thereby promoting the recovery of renal function (4), but whether stem cells can be transformed into damaged tubular endothelial cells after stem cell transplantation has been controversial. Studies have shown that the ability of transplanted stem cells to survive in the ischemic microenvironment is very limited (5). Survinvin is the smallest member of the family of inhibitors of apoptosis found in recent years and has powerful anti-apoptotic and cell-regulating functions. Therefore, in this experiment, a mouse model of renal ischemia-reperfusion injury was transplanted with MSCs transfected with Survinvin gene and EGFP-labeled. To observe the survival ability of transfected MSCs in the ischemic microenvironment and to study its mechanism of repairing renal damage.

\section{Materials And Methods}

\section{Animal and Reagents}

All procedures in this study were approved by the Animal Studies Committee of Zaozhuang Municipal Hospital (Shandong Province, China) and the experiment is in accordance with the guidelines for the care and use of laboratory animals published by the National Institutes of Health (NIH). SPF level healthy male C57BL/6 mice purchased from Saiye (Guangzhou, China) Biotechnology Co., Ltd . Cell culture medium and fetal bovine serum (FBS) were purchased from Gibco Life Technologies (Carlsbad, CA, USA). Survinvin gene recombinant lentiviral expression vector plasmid and lentiviral expression vector plasmid were purchased from Saiye (Guangzhou, China) Biotechnology Co., Ltd. BMSCs were obtained from Cyagen Biosciences, Inc. (Santa Clara, CA, USA).Creatinine, blood urea nitrogen test kits were purchased from Nanjing Jiancheng Bioengineering Institute(Nanjing, China). ELISA test kits were purchased from R\&D, USA. The experiment was completed in the laboratory of Zaozhuang Municipal Hospital.

\section{MSCs recovery and culture}

The storage tube of MSCs frozen in liquid nitrogen was taken out, placed in a $37^{\circ} \mathrm{C}$ water bath for 1 min to quickly thaw, the frozen tube was opened in a clean bench, and the frozen cells were gently blown with a pasteurized tube. And quickly transferred into a centrifuge tube containing $3 \mathrm{~mL}$ fetal bovine serum, centrifuged at $1000 \mathrm{~g}$ for 5 minutes, discard the supernatant, add $5 \mathrm{~mL}$ of F12-DMEM complete medium containing $10 \% \mathrm{FBS}$, pasteurize and paste into the cell culture flask, put Incubate in a $37^{\circ} \mathrm{C}, 5 \% \mathrm{CO} 2$ incubator. When the cell fusion reached about $90 \%$, it was digested with $0.25 \%$ trypsin, subcultured at $1: 3$ (represented as P1, P2, P3 according to the number of passages), and the culture was expanded.

\section{Construct a viral vector and transfect BMScs, detecting expression}

The empty viral vector (pLV-EX3d-P / hygro-EF1A-IRES-EmGFP, GFP-labeled vector) was purchased from Saiye (Guangzhou) Technology Co., Ltd. (Guangzhou, China). The mouse Survinvin gene was cloned into 
the pLV-EX3d-P / hygro-EF1A-IRES-EmGFP viral vector. This successfully established the pLV-EX3d-P / hygro-EF1A-mSurvivin-IRES-EmGFP viral vector. The viral vector was transfected into BMSC cultured in DMEM medium and then screened and expanded in cells. The blank pLV-EX3d-P / hygro-EF1A-IRESEmGFP vector was used to transfect cells in the control group.Two groups of passaged cell P1, P3 in the expression of EGFP is observed under an inverted fluorescence microscope.

\section{Experimental animal grouping model and specimen collection}

48 specific pathogen-free C57BL/6J mice were randomly divided into control group,ischemia reperfusion group(Marked as IR group), empty virus transfection transplantation group(Marked as MSCs

group) or survinvin gene transfection transplantation group(Marked as SVV/MSCs group). The mice were anesthetized by intraperitoneal anesthesia with $2 \%$ sodium pentobarbital solution. The mice in the control group underwent surgical incision and exposed the bilateral kidneys for $45 \mathrm{~min}$, then sutured off, and immediately injected $0.2 \mathrm{ml}$ normal saline into the tail vein. The mice in the IR group were anesthetized and exposed to bilateral renal pedicles and clamped with a non-invasive vascular clip for $45 \mathrm{~min}$. Then the clip was released and the sutured skin incision was closed. Then immediately injected $0.2 \mathrm{ml}$ normal saline into the tail vein. The mice in the MSCs group also underwent anesthesia, surgery, and bilateral renal pedicles were blocked with a non-invasive clip for $45 \mathrm{~min}$. After the blood supply was restored, the sutured skin incision was closed, and then $0.2 \mathrm{ml}$ of $1 \times 106$ MSCs infected with the self-inactive lentiviral vectorwas injected through the tail vein. The mice in the SVV/MSCs group also underwent anesthesia, surgery, and bilateral nephrons were blocked with a non-invasive clip for 45 minutes. After the blood supply was restored, the sutured skin incision was closed, and then $0.2 \mathrm{ml}$ of $1 \times 106$ survivin geneexpressing MSCs was injected through the tail vein. All mice were fed under the same conditions. Three mice were randomly selected from each group at 1 day, 3 days, 7 days, and 14 days after surgery. The blood was collected by cardiac puncture, and the serum was separated by centrifugation and stored in a refrigerator at $-20^{\circ} \mathrm{C}$. After rinsing through the heart with PBS solution, $50 \mathrm{mg}$ of right kidney tissue was excised and frozen with liquid nitrogen, and then placed in a refrigerator at $-80^{\circ} \mathrm{C}$ for use. The remaining tissue and the left kidney were fixed together with $4 \%$ paraformaldehyde for use. Use BUN and Scr test kit to detect BUN and Scr collected from the sample.『see冈fig.1》

\section{Observing the survival and differentiation of MSCs in the kidney}

The kidneys of mice were fixed by $4 \%$ paraformaldehyde for 24 hours, dehydrated by gradient ethanol, transparent with xylene, waxed and embedded in paraffin, and cut into $4 \mu \mathrm{m}$ sections. The MSCs group and SVV/MSCs group were selected on the 3rd and 14th day. Three non-continuous sections were observed under an inverted fluorescence microscope $(\times 200)$.

\section{Analysis of pathology in kidney tissue}

Ten samples were randomly selected from the bilateral kidney sections of each mouse for HE staining. Ten HE images were randomly selected from each HE slice $\times 200$ magnification. The scoring standard: 0 points $=$ normal tissue; 1 point $=$ One third of the renal tubules were damaged; 2 points $=1 / 3$ to $2 / 3$ renal 
tubule damage; 3 points $=$ more than $2 / 3$ of the renal tubules were damaged. The degree of renal tubular damage was scored as the sum of 10 random non-repetitive visual field scores in 10 sections( 0 to 300$)$.

\section{IL-10 tested by ELISA}

Take $50 \mathrm{mg}$ of the right kidney of each mouse in a $5 \mathrm{ml}$ homogenate tube, add $1 \mathrm{ml}$ of PBS, grind thoroughly for 6-8 minutes, transfer to a $1.5 \mathrm{ml} \mathrm{EP}$ tube, centrifuge at $2000 \mathrm{r} / \mathrm{min}$ for $10 \mathrm{~min}$, and take the supernatant for quantitative determination according to the ELISA kit instructions. The concentration of IL-10 in the homogenate of the kidney tissue was detected.

\section{Statistical analysis}

Experimental data were analyzed using SPSS statistical software, version 17.0 (SPSS, Inc., Chicago, IL, USA). Measurement values are expressed as the mean \pm standard error of the mean. Inter-group comparisons were performed using one-way analysis of variance. $\mathrm{P}<0.05$ was considered to indicate a statistically significant difference.

\section{Results}

\section{Results of MSCs recovery and proliferation}

The bone marrow mesenchymal stem cells that had just been resuscitated were round and single-celled. The adherent cells were seen 36 hours after resuscitation, and the cells grew vigorously on the 7th day after resuscitation, and the fusion was close to $90 \%$ (see Fig.2). The cells are typically long fusiform, swirling, and grow rapidly after passage. On the third day, the cells are covered with the bottom of the bottle.

\section{The GFP expression in MSCs and SWVMSCs}

The cells of SVV/MSCs group were transfected with pLV-EX3d-P / hygro-EF1A-mSurvivin-IRES-EmGFP gene, and the cells of MSCs group were transfected with pLV-EX3d-P / hygro-EF1A-IRES-EmGFP gene.After recovery, Two groups of passaged cell P1, P3 in the expression of EGFP is observed under an inverted fluorescence microscope. No significant decrease in fluorescence was observed in the increase in the number of passages. (See Figure 3)

\section{Test results of Mice blood BUN and Scr}

Compared with the normal group, BUN and Scr of ather groups were significantly increased at 48 hours after operation $(p<0.01$ or $p<0.05)$, and there was a downward trend with time. By the 14 th day after cell transplantation, there was no statistical differences between SVV/MSCs group and normal group. The levels of BUN and Scr in the MSCs group and the SVV/MSCs group were significantly lower than those in the IR group at the same time point.Compared with BUN and Scr levels in the MSCs group, the SVV/MSCs group decreased at 1 day, 3 days, and 7 days after cell transplantation. There was no significant 
difference between the SVV/MSCs group and the normal group at 14 days $(P>0.05)$. (See Table 1-1 and 12).

\section{The mark result of kidney pathological change and renal tubular pathological damage}

Under the HE staining of mouse kidney sections, the renal tubular lumen was dilated in the IR group after 48 hours of modeling. The renal tubular epithelial cells had edema, vacuolar degeneration, and brush-like detachment. Even exfoliated tubular epithelial cells were found in the lumen of a small number of renal tubules (see Figure 4). The damage was most significant on the second day after modeling. With the prolongation of time, the renal tubular injury of the IR group had some self-compensation repair. Compared with the IR group at the same time, in the MSCs group, the regenerated renal tubular epithelial cells with deep nuclear staining and large cell bodies were observed, and the renal tubular injury score was significantly reduced. In the SVV/MSCs group, more retinal tubular epithelial cells with deep nuclear staining and larger cell bodies were found, and the degree of tubular damage was significantly reduced compared with the previous two groups. By the 14th day, the tissue structure was close to normal. (See Table 2).

\section{The result of BMSCs survival and differentiation}

After cell transplantation, MSCs expressing EGFP were mainly distributed around the glomerulus, the inner wall of small blood vessels, the interstitial space between the renal tubules and the renal tubules, and almost no EGFP-expressing MSCs were found on the inner wall of the renal tubules. (See Figure 5)

\section{IL-10 level of kidney tissue of mice in each group}

It can be seen that in the IR group, the level of IL-10 gradually decreased with the progression of the disease, while the level of IL-10 in the MSCs group gradually increased with the progression of the disease, peaked at about 7th day, and then decreased. The IL-10 levels in the MSCs group and the SVV/MSCs group were significantly higher than those in the control group and the IR group $(P<0.01$ or $P<0.05)$. The level of IL-10 in the SVV/MSCs group increased rapidly with the progress of the disease, reached a peak in about 3th day, and then decreased. The IL-10 level in the SVV/MSCs group was significantly higher than that in the MSCs group at the same time $(P<0.01$ or $P<0.05)$. (See Table3 ).

\section{Discussion}

Currently, the role of stem cell transplantation in renal ischemic injury has been confirmed by many scholars. Such as the use of cisplatin to build a rat model of acute renal failure Morigi (6), the study found that application of MSCs can protect a fellow female rats tubular necrosis and renal dysfunction, of P. Semedo(7) using MSCs treatment of renal ischemia injury rat model, results showed that $24 \mathrm{~h}$ after infusion of MSCs plasma urea and creatinine decreased significantly, simultaneous renal cell regeneration also significantly enhanced can be seen from the results of this experiment, the MSCs in the repair of renal function in this role to support the former view, the day after stem cell transplantation 
MSCs group and the S / MSCs group of Scr and BUN levels decreased renal pathological damage reduced than the IR group, the kidney tissue of HGF, bFGF and IL-10 levels significantly higher than the IR group, but the decrease levels of Scr and BUN, the extent of the renal tubular damage has been mitigated, and protective factors within the kidney increased, the S / MSCs group more obvious than the MSCs group, From this we see that the same amount of cell transplantation in the S / MSCs group is more conducive to the recovery of renal function in mice after ischemic injury. In mouse kidney cells alive for 3 days and 14 days for observation and analysis, we found that the number of viable cells of three days or 14 days of the S / MSCs group is much larger than the MSCs group, which may be MSCs the by Survinvin gene transfection, its anti-apoptotic ability are closely related.

Survivin is a new member of inhibitor of apoptosis protein (Inhibitorofapoptosis IAP) family, is found the strongest inhibitor of apoptosis through the interaction of CDK4 to form complexes, so that freed from the complex combination of CDK4 p21waf1/cip1 and mitochondria of procaspase-3 interaction, start the inactivation of caspase-3, thereby inhibiting Fas-mediated apoptosis(8). Shin etc(9) the use of vitro caspase inhibition studies confirmed and Survivin could directly inhibit caspase- 3 and caspase-7, thereby blocking the apoptotic process. Conway, the EM (10) confirmed that increased Survivin expression in cerebral ischemia model can promote ischemic tissue revascularization, thus contributing to the recovery of brain function, but have the same role of survivin in the renal ischemia model, the current domestic outside yet to see the related research reports.

The role of MSCs in repair after renal ischemia injury have no doubt, but due to the direct killing effect on the transplanted cells of ischemia-reperfusion injury in the organization, as well as a series of inflammatory cascade reflects the increase in oxygen free radicals, a large number of proapoptotic cells factors and chemical factors exist, making the simple cells of stem cell transplantation is very limited ability to survive in the microenvironment after ischemia(11) of MSCs after transplantation play a therapeutic effect of the number of cells is drastically reduced. This experimental application of a strong anti-apoptotic effect of Survivin gene transfected MSCs transplanted into the mice ischemia-reperfusion injury model, found that regardless of the 3 days or 14 days of the S / MSCs group survival in the kidney cells is far higher than the number of pure MSCs transplantation group, so we speculate that transfected Survivin gene, through a powerful anti-apoptotic ability to increase the viability of MSCs in the ischemic microenvironment.

After renal ischemia-reperfusion injury, edema and necrosis of the main pathological changes of renal tubular endothelial cells, and thus seriously affect renal function(12). The traditional view is that, after transplantation of MSCs into the blood circulation, the effect of the damage the kidneys secrete chemokines, adhesion molecules such as homing to kidney damage area characteristics $(13,14)$, and located in the region of the kidney damage differentiates into renal tubular epithelial cells thus contributing to renal repair. Controversial but a transplanted MSCs can differentiate renal tubular endothelial cells Togel, etc(15) CDFA and Y chromosome markers, MSC transplantation in the SD rat ischemia-reperfusion model, found only in the MSC 2 hours after transplantation, 24h can be detected in the kidney to the CFDA mark of MSCs, $72 \mathrm{~h}$ after undetectable the CFDA mark of MSCs. And $\mathrm{Y}$ 
chromosome markers of MSCs detected in 24 after less than we found in the observation for three days and 14 days mouse renal cells expressing EGFP 3 days, 14 days of MSCs express FGFP mostly located in the glomerular around the inner wall of small blood vessels, and tubular and tubular interstitial site in the tubular wall almost find no expression of EGFP of MSCs, indicating that the transplantation of MSCs can be anchored, lives in a damage kidney, but not like the traditional view that as to the injury of renal tubular endothelial cell transformation indicate that MSCs are other ways to repair damage the kidneys. With the development of research on stem cell transplantation, the specific mechanism of MSCs for repairing of damaged kidneys has also emerged. Studies have shown that MSCs secretion mechanism in the repair of renal injury plays a major role $(16,17)$, this view has gain the recognition and support of the majority of scholars. Florian Togel et al (18) demonstrated that some renal protective factors, such as bFGF, IL-10, and IGF-1, were significantly elevated in the renal ischemia-reperfusion injury model of transplanted MSCs, and the paracrine effect of MSCs was on acute ischemic renal injury. The role of repair in the repair of IL-10 as an anti-inflammatory factor in acute ischemic kidney injury has been well known (19, 20). From the results of IL-10 detection and cell survival in each group of mice, it can be seen that the IL10 level of MSCs after transplantation is significantly higher than that of the IR group. This result is related to the results of the aforementioned Florian Togel et al. Similarly, the S/MSCs group transfected with Survivin gene not only increased the number of surviving cells, but also increased the level of protective factor IL-10 after renal ischemic injury compared with IR and MSCs. $(P<0.01$ or $p<0.05)$, and there was no statistical difference between the pathological changes on day 14 and the normal control group.This indicates that SVV/MSCs transfected with Survivin gene not only enhances their antiapoptotic ability, but also increases the number of viable cells in the ischemic kidney, increases the secretion of protective factor of the kidney, and promotes the repair of kidney function and structure.

\section{Conclusion}

In summary, although MSCs transplantation can play a role in the repair of post-ischemic kidneys, the survival of MSCs in the microenvironment after renal ischemia-reperfusion injury is severely limited, and the number of therapeutic cells is also Significantly reduced. However, MSCs transfected with Survivin gene not only enhanced their anti-apoptotic ability, but also increased the number of viable cells, and further increased the secretion of renal protective factors, promoting renal function and structural repair. In the results of observing the localization and differentiation of cells after transplantation and the detection of IL-10 levels in the kidneys of each group of mice, we did not observe EGFP-expressing MSCs on the inner wall of the renal tubules. This suggests that the pathway by which MSCs function is achieved through its paracrine effects, rather than directly to the renal tubular epithelial cells.

However, there are still two unanswered questions in our experimental study: First, whether Survivin produced by MSCs transfected with Survivin gene can directly exert anti-apoptotic effect on renal tubular epithelium; Second, Survivin Whether the increase in expression can promote revascularization in the kidney as in the cerebral ischemia model, thereby promoting the recovery of renal function, remains to be confirmed by further research. 


\section{Declarations}

\section{Ethics approval and consent to participate}

The animal experiment of this study have been approved by the Ethic committee of Zaozhuang municipal hospital.

\section{Consent for publication}

Not applicable.

\section{Availability of data and material}

Not applicable.

\section{Competing interests}

The authors declare that they have no competing interests.

\section{Funding}

Not applicable.

\section{Authors' contributions}

Qianqian Wang designed this study. Xiaofeng Yang performed experiments. Xiaofeng Yang made statistical analysis. Qianqian Wang reviewed manuscript. All authors approved final manuscript.

\section{Acknowledgements}

We feel grateful to all the support from our experimenters.

\section{References}

1. Liu, N. , Wang, H. , Han, G. , Cheng, J. , Hu, W. , \& Zhang, J. . (2018). Enhanced proliferation and differentiation of ho-1 gene-modified bone marrow-derived mesenchymal stem cells in the acute injured kidney. International Journal of Molecular Medicine. doi:10.3892/ijmm.2018.3670.

2. Kankuri, E. , Elina E. Mervaala, Storvik, M. , Aija M.J. Ahola, Levijoki, J. , \& Dominik N. Müller, et al. (2015). Exacerbation of acute kidney injury by bone marrow stromal cells from rats with persistent renin-angiotensin system activation. Clinical Science, 128(11), 735-747. doi:10.1042/CS20140445.

3. La Manna, G. , Bianchi, F. , Cappuccilli, M. , Cenacchi, G. , Tarantino, L. , \& Pasquinelli, G. , et al. (2011). Mesenchymal stem cells in renal function recovery after acute kidney injury: use of a differentiating agent in a rat model. CELL TRANSPLANTATION, 20(8), 11931208. doi:10.3727/096368910x543394 
4. Kale, S. , Karihaloo, A. , Clark, P. R. , Kashgarian, M. , Krause, D. S. , \& Cantley, L. G. . (2003). Bone marrow stem cells contribute to repair of the ischemically injured renal tubule. Journal of Clinical Investigation, 112(1), 42--49. doi: 10.1172/JCI200317856.Introduction

5. Mylotte, L. A. , Duffy, A. M. , Murphy, M. , O'Brien, T. , \& Szegezdi, E. . (2008). Metabolic flexibility permits mesenchymal stem cell survival in an ischemic environment. Stem Cells, 26(5), 1325-1336. doi:10.1634/stemcells.2007-1072

6. Morigi M, Imberti B, Zoja C, et al. Mesenchymal stem cells are renotropic, helping to repair the kidney and improve function in acute renal failure. J Am Soc Nephrol , 2004,15: 17941804.doi:10.1097/01.ASN.0000128974.07460.34

7. P. Semedo, P.M. Wang, T.H. Andreucci,et al. Mesenchymal Stem Cells Ameliorate Tissue Damages Triggered by Renal Ischemia and Reperfusion Injury. Transplantation Proceedings, 2007, 39: 421423.doi:10.1016/j.transproceed.2007.01.036

8. Martínez-García, David, Manero-Rupérez, Noemí, Quesada, R. , Korrodi-Gregório, Luís, \& Soto-Cerrato, V. . (2018). Therapeutic strategies involving survivin inhibition in cancer. Medicinal Research Reviews. doi:10.1002/med.21547

9. Shin S, Sung BJ, Cho YS, et al. An anti-apoptotic protein human survivin is a direct inhibitor of caspase-3 and -7.Biochemistry, 2001, 40(4):1117-1123.doi:10.1021/bi001603q

10. Conway EM, Zwerts F, Van Eygen V, et al. Survivin-dependent angiogenesis in ischemic brain: molecular mechanisms of hypoxia-induced up-regulation.Am J Pathol,2003 ,163(3):935946.doi: 10.1016/s0002-9440(10)63453-0

11. Zhang, T. , Yang, X. , Liu, T. , Shao, J. , Fu, N. , \& Yan, A. , et al. (2017). Adjudin-preconditioned neural stem cells enhance neuroprotection after ischemia reperfusion in mice. Stem Cell Research \& Therapy, 8(1), 248. doi: 10.1186/s13287-017-0677-0

12. Gerber, W. E. G. , Cesar, F. J. , Gregori, C. N. , Osvaldo, M. , Cesar, K. F. , \& Cesar, M. T. , et al. (2017). Tadalafil protector effect during ischemia-reperfusion in rats. Acta Cirurgica Brasileira,32(11), 973983. doi: 10.1590/s0102-865020170110000009

13. Adriana López Ponte, Marais, E. , Gallay, N. , Alain Langonné, Delorme, B. , \& Olivier Hérault, et al. (2007). The in vitro migration capacity of human bone marrow mesenchymal stem cells: comparison of chemokine and growth factor chemotactic activities. Stem Cells (Miamisburg), 25(7), 1737-1745. doi:10.1634/stemcells.2007-0054

14. Togel, F. , Isaac, J. , Hu, Z. , Weiss, K. , \& Westenfelder, C. . (2005). Renal sdf-1 signals mobilization and homing of cxcr4-positive cells to the kidney after ischemic injury. Kidney International, 67(5), 1772-1784.doi:10.1111/j.1523-1755.2005.00275.x

15. Florian Tögel, Hu, Z. , Weiss, K. , Isaac, J. , Lange, C. , \& Westenfelder, C. . (2005). Administered mesenchymal stem cells protect against ischemic acute renal failure through differentiationindependent mechanisms. Am J Physiol Renal Physiol, 289(1), F31. doi:10.1152/ajprenal.00007.2005 
16. Yuan X, Li D, Chen X, Han C, et al.Extracellular vesicles from human-induced pluripotent stem cellderived mesenchymal stromal cells (hiPSC-MSCs) protect against renal ischemia/reperfusion injury via delivering specificity protein (SP1) and transcriptional activating of sphingosine kinase 1 and inhibiting necroptosis.Cell Death Dis. 2017 Dec 11;8(12):3200. doi: 10.1038/s41419-017-0041-4.

17. Li, Y. , Liu, J. , Liao, G. , Zhang, J. , Chen, Y. , \& Li, L. , et al. (2018). Early intervention with mesenchymal stem cells prevents nephropathy in diabetic rats by ameliorating the inflammatory microenvironment. Int J Mol Med. 2018 May;41(5):2629-2639. doi: 10.3892/ijmm.2018.3501. Epub 2018 Feb 16.

18. Togel F, Weiss K, Yang Y, et al. Vasculotropic, paracrine actions of infused mesenchymal stem cells are important to the recovery from acute kidney injury. Am J Physiol Renal Physiol. 2007, 292: F1626-F1635.doi: 10.1152/ajprenal.00339.2006

19. Rifkin, G. D. , Pintucci, G. , Klein, M. S. , \& Bikfalvi, A. . (1997). Biological roles of fibroblast growth factor-2. Endocrine Reviews, 18(1), 26. doi: 10.1210/edrv.18.1.0292

20. Deng, J. , Kohda, Y. , Chiao, H. , Wang, Y. , Hu, X. , \& Hewitt, S. M. , et al. (2001). Interleukin-10 inhibits ischemic and cisplatin-induced acute renal injury. Kidney International, 60(6), 2118-2128. doi:10.1046/j.1523-1755.2001.00043.x

\section{Tables}

Table 1-1 Comparison of the kidney function index in each group(BUN:mmol/L, mean \pm standard deviation)

\begin{tabular}{|c|c|c|c|c|}
\hline Groups & Day & Day 3 & Day & Day 14 \\
\hline Normal control group & 6.300 .61 & 6.200 .41 & 6.400 .33 & 6.450 .31 \\
\hline IR group & $21.740 .87^{\star}$ & $16.352 .87^{\star}$ & $11.320 .22^{\star}$ & $10.740 .53^{\star}$ \\
\hline MSCs group & $14.631 .63^{*} \#$ & 13.061 .23 * \# & 10.610 .44 * \# & 9.760 .36 * \# \\
\hline SVV/MSCs group & $13.261 .69 * \# \mathbf{\Delta}$ & $9.101 .23^{*} \# \boldsymbol{\Delta}$ & 8.540.64*\# & $6.920 .66^{* \star \#}$ \\
\hline
\end{tabular}

${ }^{\star P}<0.05,{ }^{* \star P} \otimes 0.05$ vs. Normal control group;

$\# \mathrm{P}<0.05$ vs. I R group;

$\Delta P<0.05$ vs.MSCs group.

Table 1-2 Comparison of the kidney function index in each group(Scr: $\mu \mathrm{mol} / \mathrm{L}$, mean \pm standard deviation) 


\begin{tabular}{|lclll|}
\hline Groups & Day 1 & Day 3 & Day 7 & Day 14 \\
\hline Normal control group & 25.693 .54 & 26.612 .54 & 25.103 .31 & 25.582 .54 \\
\hline IR group & $52.333 .93^{*}$ & $44.922 .15^{\star}$ & $44.683 .29^{*}$ & $43.583 .28^{\star}$ \\
& & & & \\
\hline MSCs group & $42.644 .81^{*} \#$ & $39.002 .54^{\star} \#$ & $38.414 .71^{*} \#$ & $35.613 .09^{\star} \#$ \\
\hline SVV/MSCs group & $32.570 .82^{\star} \# \triangle$ & $31.411 .18^{\star} \# \triangle$ & $31.251 .14^{\star} \# \triangle$ & $26.93 .55^{\star \star} \# \triangle$ \\
\hline
\end{tabular}

${ }^{\star} \mathrm{P}<0.05,{ }^{* \star} \mathrm{P} \otimes 0.05$ vs. Normal control group;

$\# \mathrm{P}<0.05$ vs. I R group ;

$\triangle P<0.05$ vs.MSCs group.

Table2 Comparison of scores of renal tubular damage in mice (mean \pm standard deviation).

\begin{tabular}{|c|c|c|c|c|}
\hline Groups & Day 1 & Day 3 & Day & Day 14 \\
\hline Control group & 00 & 00 & 00 & 00 \\
\hline IR group & $267.8317 .27^{\star}$ & $256.507 .55^{\star}$ & 180.5012.17* & $97.336 .41^{\star}$ \\
\hline MSCs group & $131.1712 .2^{\star} \#$ & 109.177.81*\# & $90.337 .89 * \#$ & $53.178 .17 * \#$ \\
\hline SVV/MSCs group & $112.839 .75^{\star} \# \boldsymbol{\Delta}$ & $93.507 .06 * \# \mathbf{\Delta}$ & $72.677 .34 * \# \mathbf{\Delta}$ & 32.835.85*\# \\
\hline
\end{tabular}

Vs. normal group, $* P<0.01$;

Vs. IR group, $\# P<0.01$;

Vs. MSCs, $\triangle P<0.01$

Table3 Comparison of kidney tissue IL-10 levels in each group ( $\mathrm{ng} / \mathrm{L}$.mean \pm standard deviation).

\begin{tabular}{|c|c|c|c|c|}
\hline Groups & Day & Day 3 & Day & Day 14 \\
\hline Normal control group & $53.84 \pm 4.84$ & $53.09 \pm 3.34$ & $56.21 \pm 1.92$ & $52.96 \pm 3.84$ \\
\hline IR group & $65.72 \pm 1.76^{*}$ & $63.09 \pm 1.32^{\star}$ & $60.15 \pm 1.20 *$ & $57.15 \pm 2.51$ * \\
\hline MSCs group & $95.81 \pm 4.31 * \#$ & $78.56 \pm 3.21 * \#$ & $84.06 \pm 2.15^{\star} \#$ & $65.01 \pm 3.24^{\star} \#$ \\
\hline SVV/MSCs group & $84.76 \pm 3.41^{*} \# \triangle$ & $90.12 \pm 3.41^{*} \# \triangle$ & $89.76 \pm 4.41^{\star} \# \triangle$ & $80.76 \pm 3.41 * \# \triangle$ \\
\hline
\end{tabular}


${ }^{*} \mathrm{P}<0.05$ vs. Normal control group;

\#P<0.05 vs. I R group ;

$\triangle P<0.05$ vs. MSCs group.

\section{Figures}

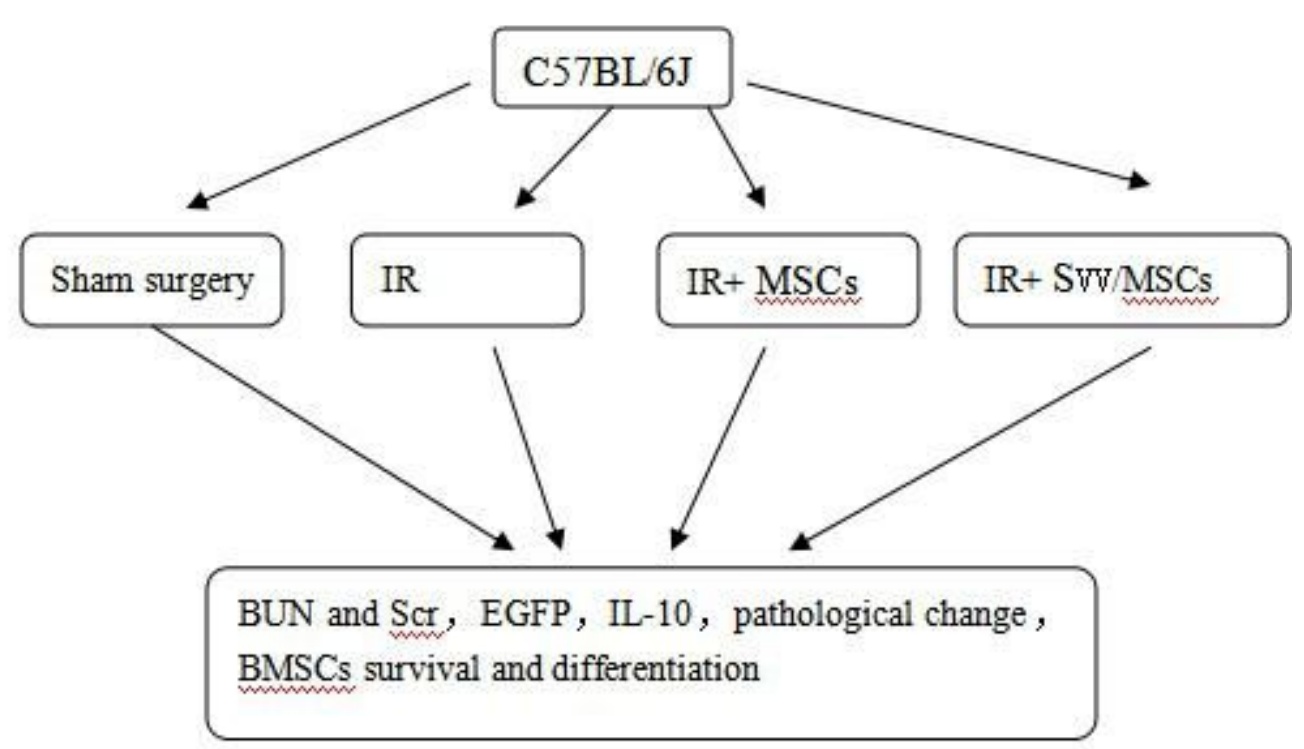

\section{Fig.1 Experimental flow diagram}

\section{Figure 1}

Experimental flow diagram. 48 specific pathogen-free C57BL/6J mice were randomly divided into control group,ischemia reperfusion group(Marked as IR group), empty virus transfection transplantation group(Marked as MSCs group) or survinvin gene transfection transplantation group(Marked as SVV/MSCs group). 

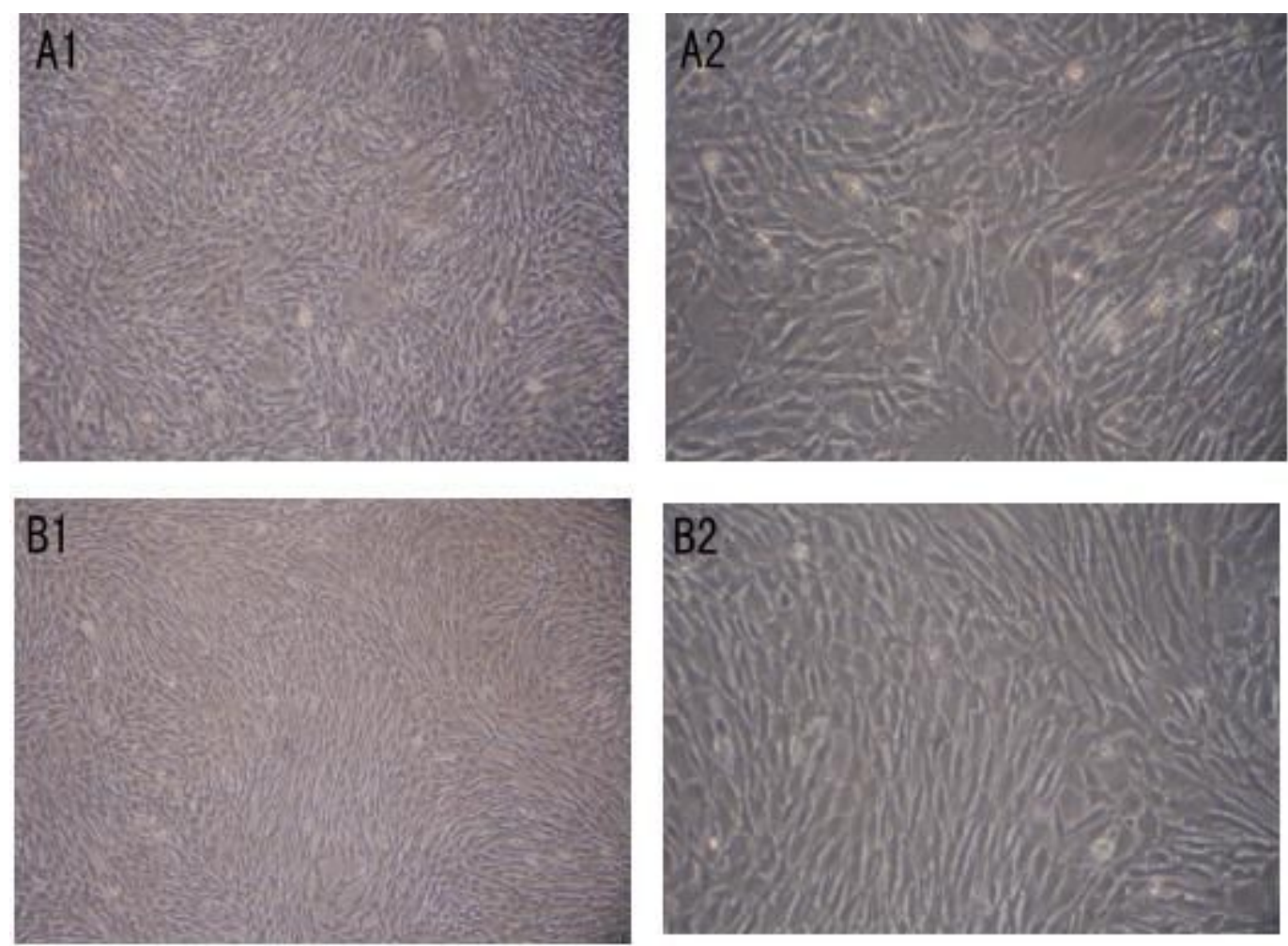

Fig 2 Morphologic Observation of MSCs by microscope

(A1) 7 daysafterrecoven, M5Cs group, $\times 100 \quad(A 2) 7$ da 15 afterreconen, M5Cs group, $\times 200$

(B1) 7 days afterrecoveny, 5W/M5Csgroup , $\times 100 \quad$ (B 2) 7 da ys after recowen, 5W/MSCs group $\times 200$

\section{Figure 2}

Morphologic Observation of MSCs by microscope. Recovered and inoculated Bone Marrow-derived Mesenchymal Stem Cells shows a round, single-cell distribution and recovered cells are visible adherent cells after $36 \mathrm{~h}$ and after 7 days of recovery, the cells grow well the integration is up to $90 \%$ 

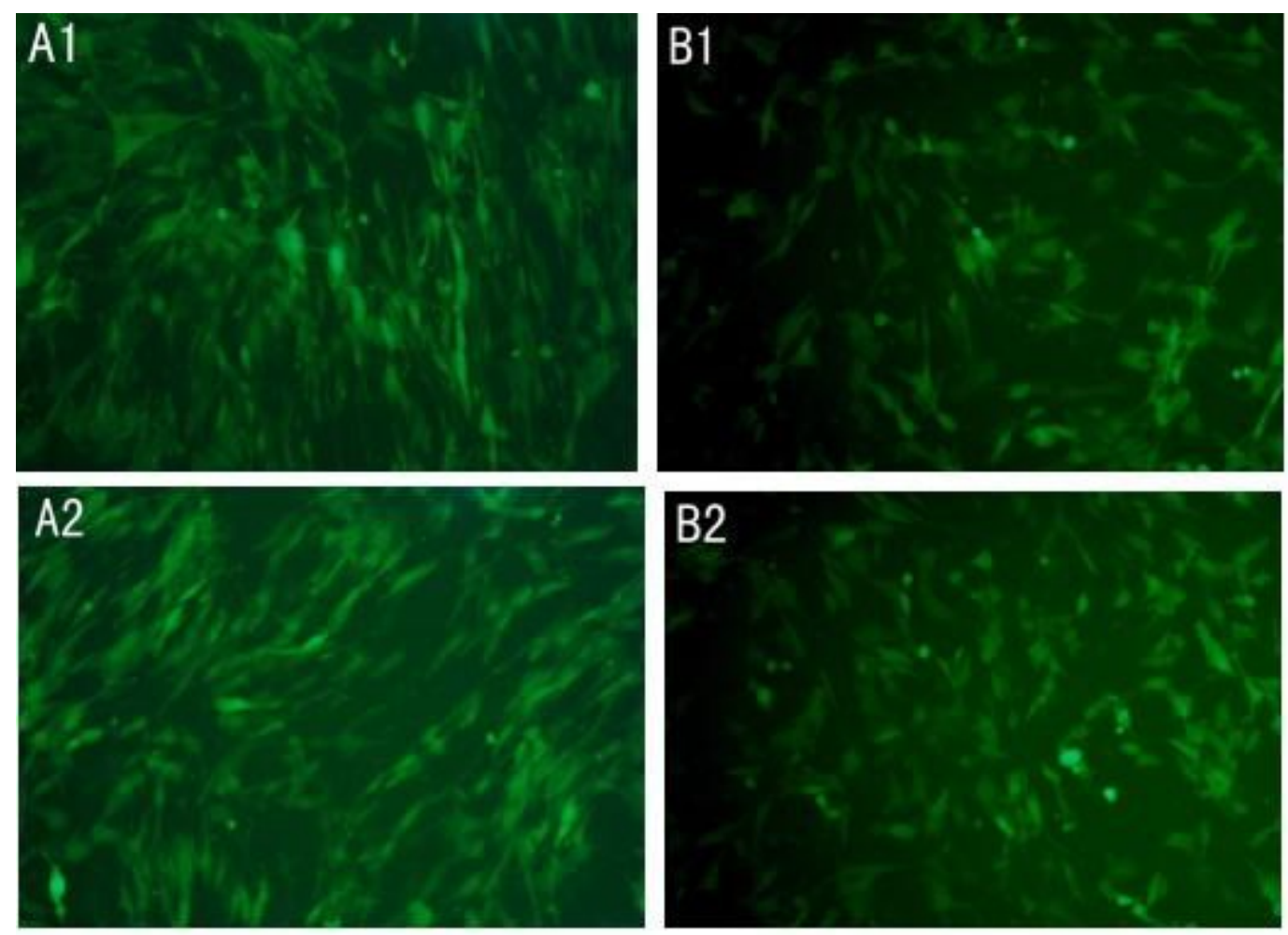

Fig.3 Exp ression of green fluoresc ent prot ein of MSCs by Inverted fluorescence mic roscope

(A1) EGFP expression of P1 cells in MSCs group

(A2) EGFP expression of $P 3$ cells in MSCs group

(B1) EGFP expression of P1 cells in SW/MSC s group

(B2) EGFP expression of $P 3$ cells in SW/MSCs group

\section{Figure 3}

Expression of green fluorescent protein of MSCs by Inverted fluorescence. After recovery, two groups of cells p1, p3, visible green fluorescence is observed by an inverted fluorescence microscope, no significant fluorescence decrease with the increase in the number of passages. 


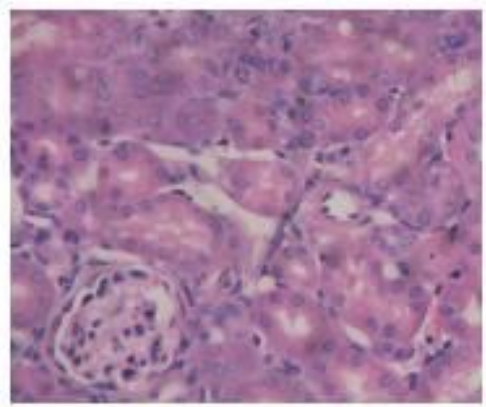

IR 组

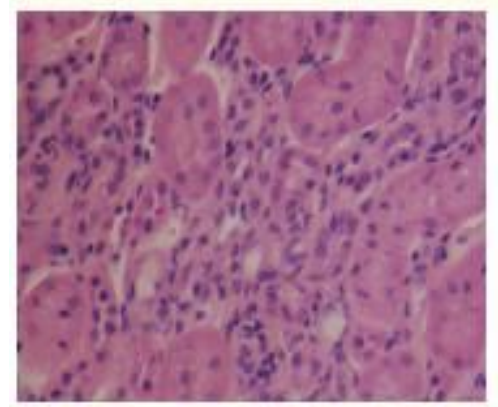

IR 组.

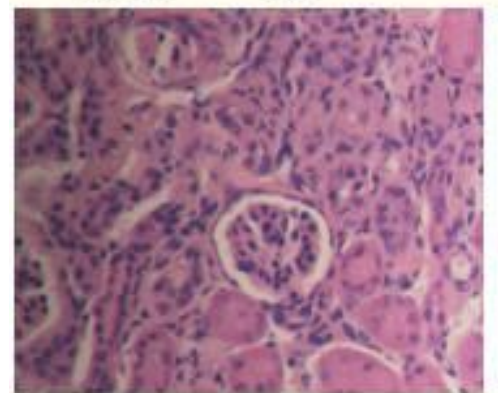

IR 组

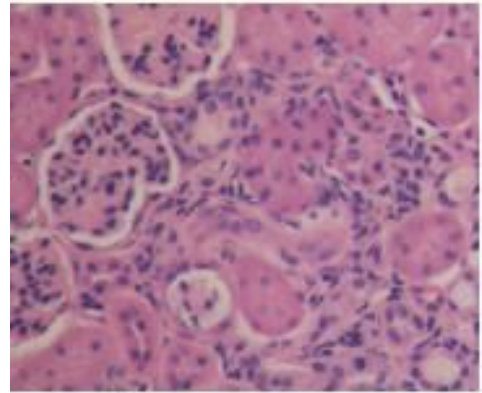

IR 组

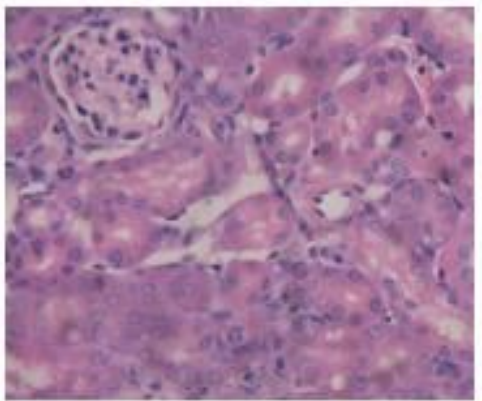

MSC5 组 $1 d$

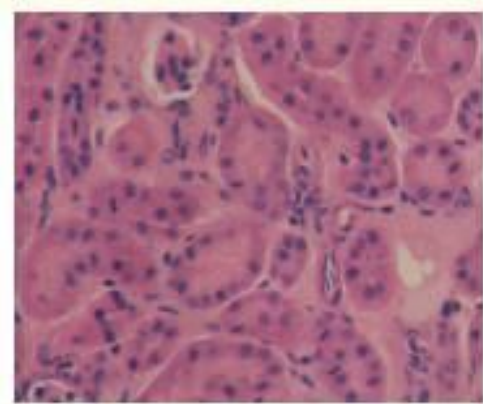

MSC5 组 $\quad 3 d$

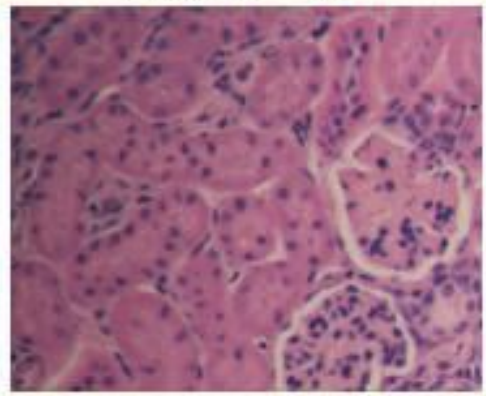

MSC5 组

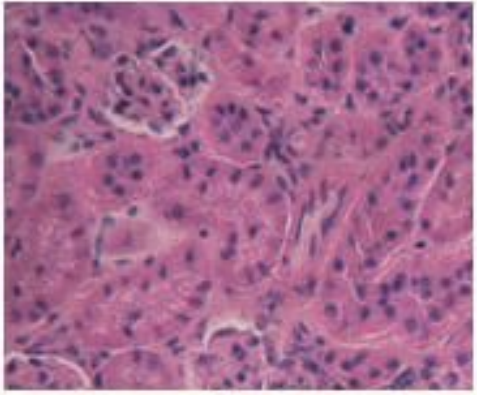

MSC5 组 14d

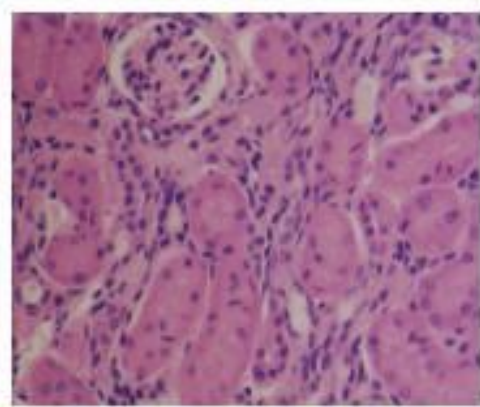

SVV/MSC5 组 1d

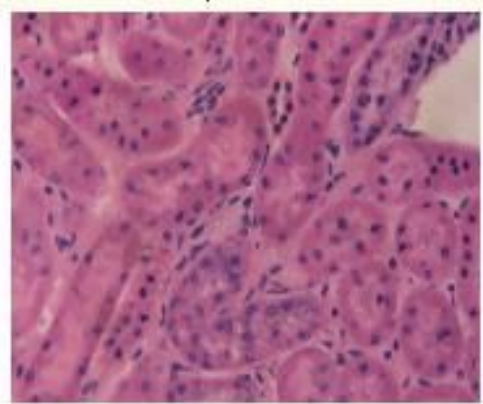

SVV/MSC5 组 3d

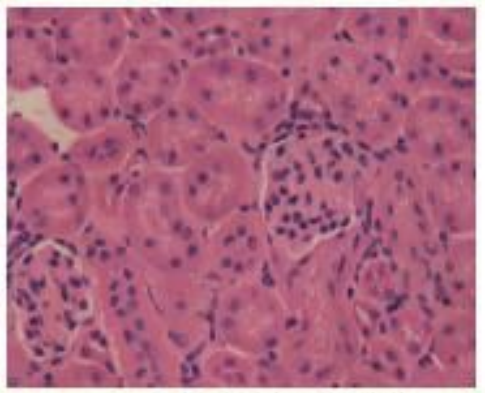

SVV/MSC5 组. 7d

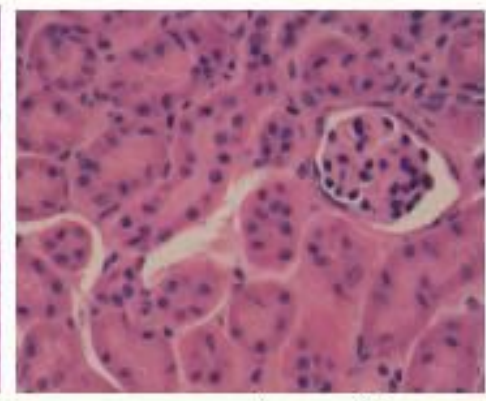

SW/MSC5 组 $14 \mathrm{~d}$

Fig.4 Kidneys of each group with hematoxylin and eosin $(\times 200)$

\section{Figure 4}

Kidneys of each group with hematoxylin and eosin $(\times 200 \rrbracket$ Under the HE staining of mouse kidney sections, the renal tubular lumen was dilated in the IR group after 48 hours of modeling. The renal tubular epithelial cells had edema, vacuolar degeneration, and brush-like detachment. Even exfoliated tubular epithelial cells were found in the lumen of a small number of renal tubules. 

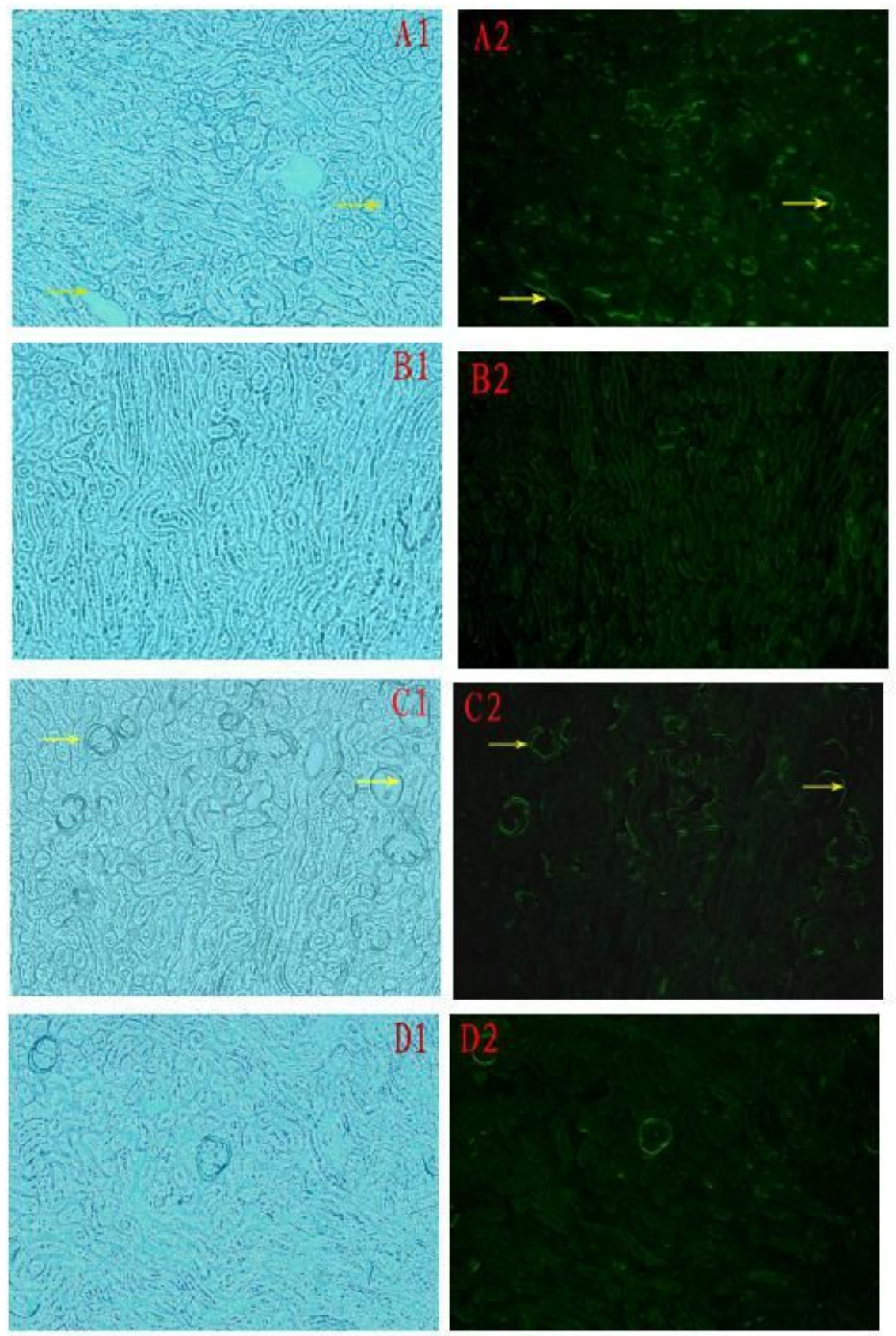

Fig. 5 Im age of Kichey after cell transplant by Inverted fluorescence microscope

(A) SW/MSCs group 3d (B) MSCs group 3d

(C) SW/MSCs group $14 \mathrm{~d}$ (D) MSCs group $14 \mathrm{~d}$

(A1-D1) for renal biopsy in the ordinary light im age

(A2-D2), the transplanted cells under the microscope the same field of green fluorescent expression of images

\section{Figure 5}

Image of Kidney after cell transplant by Inverted fluorescence microscope. After cell transplantation, MSCs expressing EGFP were mainly distributed around the glomerulus, the inner wall of small blood vessels, the interstitial space between the renal tubules and the renal tubules, and almost no EGFPexpressing MSCs were found on the inner wall of the renal tubules. 